\title{
NIVELES DE DISFUNCIÓN FAMILIAR, EN VEINTE MUJERES VÍCTIMAS DE VIOLENCIA INTRAFAMILIAR EN EL MUNICIPIO DE ARMENIA
}

FAMILY DYSFUNCTION LEVELS IN TWENTY WOMEN, VICTIMS OF INTRAFAMILY VIOLENCE, IN THE MUNICIPALITY OF ARIMENIA.

Recibido: febrero de 2013 - Revisado: agosto de 2013 - Aceptado: 30 de octubre de 2013

Por: Juan Gonzales Portillo; Francia Merly Rivas Marin ${ }^{2}$;

Ximena Alexandra Marin Restrepo ${ }^{3}$, Leidy Viviana Villamil Bonilla. ${ }^{4}$

\section{RESUMEN.}

Esta investigación pretende encontrar posibles niveles de disfunción familiar en las familias de 20 mujeres víctimas de violencia intrafamiliar en la ciudad de Armenia - Quindío, para lo cual se aplicó el APGAR familiar, el cual evalúa la satisfacción respecto a 5 variables: adaptación, participación, ganancia o crecimiento, afecto y recursos. El estudio encontró que las familias presentan disfunción leve $(35 \%)$, moderada $(30 \%)$ y severa $(20 \%)$, mientras un porcentaje muy bajo tiene buena función familiar (15\%); lo anterior indica una correlación importante entre violencia intrafamiliar y disfunción familiar, condición que disminuye la calidad de vida del núcleo familiar, alterando los roles, los modos de expresión afectiva y la interiorización de lo normativo.

\section{PALABRAS CLAVE.}

APGAR familiar, autopoiesis, función familiar, disfunción familiar, violencia intrafamiliar, psicología, sistema, sistémico.

\section{ABSTRACT.}

This research aims to find possible levels of family dysfunction in families of 20 women victims of domestic violence in the city of Armenia - Quindio, which applied the family Apgar. It evaluates satisfaction with respect to 5 variables: adaptation, participation, gain or growth, affection and resources. The study found that the 35\% families have mild dysfunction, 30\% moderate dysfunction, and $20 \%$ severe dysfunction, while a very low percentage has good family function, which represents $15 \%$. This indicates a significant correlation between intrafamily violence and family dysfunction, a condition that reduces the family unit's quality of life, altering roles, modes of emotional expression, and the internalization of norms.

\section{KEY WORDS AND EXPRESSIONS.}

Family Apgar score; Autopoiesis, Family function; Family dysfunction; Intrafamily violence; Psychology; System; and Systemic.

${ }^{1}$ Psicólogo especialista. Investigador principal. Coordinador del programa de psicología de la Universidad de San Buenaventura Seccional Medellín - Convenio con la Fundación Universitaria San Martín extensión Ibagué. Colombia 2011. Email: juancgp9@hotmail.com.

${ }^{2}$ Licenciada en Matemáticas y Especialista. Docente Investigadora del programa de Psicología de la Universidad de San Buenaventura Medellín. Email: franciamerly@gmail.com.

${ }^{3}$ Estudiante de sexto semestre del programa de Psicología de la Universidad de San Buenaventura Medellin. Email: ximemarin20@hotmail.com.

${ }^{4}$ Estudiante de sexto semestre del programa de Psicología de la Universidad de San Buenaventura Medellin. Email: villamileidyb@hotmail.com. 


\section{Introducción.}

El primer estudio sobre la violencia doméstica lo realizó la Organización Mundial de la Salud (OMS) en el año 2005, en él es descrita como aquella violencia ejercida por la pareja afirmando que es la forma de violencia más común en la vida de las mujeres, presentándose con mayor frecuencia que las agresiones o violaciones perpetradas por extraños o conocidos. La entidad la ubica en el plano de un problema social de amplio espectro ya que, afecta a las personas en su salud fisica y psicológica de manera global y tiene como consecuencia problemas biopsicosociales permanentes para el individuo y la familia afectada. Para el Instituto Nacional de Medicina Legal y Ciencias Forenses (2010), la violencia doméstica "es toda aquella acción u omisión que puede generar lesión física o psicológica, entre los victimarios a cualquier persona del grupo familiar" (p.101), en ella se incluyen como victimas adultos mayores, mujeres, niños, niñas y adolescentes, mientras los victimarios son cuidadores y cualquier otra persona que ejerza sobre la víctima algún tipo de relación de poder.

El estudio de la violencia intrafamiliar (VIF) implica el análisis de las causas y consecuencias cada vez más complejas, pues el modo como altera la institución familiar instaura un problema de seguridad ciudadana y de descomposición del tejido comunitario que preocupa a todas las instituciones de la sociedad. De acuerdo con Sagot \& Carcedo (2008) en América la violencia intrafamiliar afecta a una de cada tres mujeres, lo cual la instaura la VIF en el ámbito de los actos de lesa humanidad, las discriminación y la inequidad de género. La VIF es una de las problemáticas que a nivel nacional impacta la dinámica individual, familiar y social de las comunidades, los datos presentados por el Instituto Nacional de Medicina Legal y Ciencias Forenses (INMLCF-Forensis) indican que los casos pasaron de 77.745 (2007) a 89.803 (2008), mostrando un incremento del 16\% en los eventos. Es importante mencionar que la vivienda fue el lugar donde se produjo el mayor número de agresiones y presentó un total de 55.677 casos (Forensis. 2009, pp.112-113). En el departamento del Quindío al 30 de octubre de 2009 se reportaron 1.511 casos de violencia intrafamiliar contra miembros familiares, de ellos 1.110 eventos fueron sobre mujeres (Defensoría del pueblo. 2010). Así mismo el $76 \%$ de las mujeres maltratadas fisicamente no acude a ninguna parte para denunciar la agresión de la que fue objeto (Profamilia, Cap. XIII, Bogotá, 2005).

Para medicina legal la descripción del comportamiento de la violencia intrafamiliar en el 2010 mostró a nivel nacional un total de 89.436 registros de violencia intrafamiliar, " 4.426 menos que en el 2009, año en el que se conocieron 93.862. La tasa nacional fue de 196,53 por cada cien mil habitantes" (FORENSIS. 2010, p.100). De acuerdo a la institución la VIF estuvo connotada en su mayoría por la violencia de pareja, hecho que alcanzó cifras del 64,7\%; seguida de la violencia entre otros familiares $(18,1 \%)$, la violencia a niños, niñas y adolescentes $(15,4 \%)$ y la violencia al adulto mayor con el $1,8 \%$. Las mujeres son víctimas frecuentes de VIF (78\% en el 2010) por lo que resulta importante mencionar que la violencia de pareja deja cada año un número mayor de víctimas especialmente de mujeres. En el año 2010 "el 88,5\% de las víctimas fueron mujeres. Sin embargo, a partir de los 65 años esa diferencia se hace menor" (p.113) con un rango de edad prevalente entre de los 25 a los 29 años $(23 \%)$. En el género femenino en cuanto la edad al momento de la agresión, el rango de los 20 a los 24 es el más vulnerable. La agresión fue perpetrada en el $41 \%$ de los casos por sus compañeros permanentes, seguido por los esposos (as) (25\%) y los ex compañeros (as) permanentes $(16,3 \%)$ 
De acuerdo con Forensis (2007) la violencia intrafamiliar por ser del ámbito privado, de la misma manera que la violencia sexual, no está del todo dimensionada debido al subregistro de denuncias no presentadas por diferentes motivos, casi siempre presentes en la víctima. En este sentido Andrade. J y Valencia. L (2011) encontraron que las víctimas procedian de hogares violentos en el que uno de sus padres consumía alcohol y aunque esto no es una condición sine qua non de todos los actos de VIF, se puede precisar como un factor determinante de la disfuncionalidad del núcleo familiar. Análogamente la VIF se instauró en las mujeres de escasos recursos económicos sin antecedentes de VIF, ni consumo de alcohol de sus padres; en estos escenarios los actores violentos (padres, hermanos, parejas) y los modos disfuncionales como comunican la protección, el dominio o la coerción, se constituyeron en formas mediáticas de comunicación intrafamiliar, estos factores como condiciones de riesgo son útiles para la reproducción de la violencia en escenarios intrafamiliares, y se asocian a condiciones psicosociales de las víctimas tales como, una educación formal incompleta, patrones disfuncionales de relación, dependencias con el agresor a todo nivel y el nivel de presión patriarcal de los grupos locales, muchas mujeres se ven presionadas por grupos armados que de acuerdo con el ACNUR (2008) dan continuidad "al reclutamiento de menores, actos de violencia sexual contra mujeres y niñas" (p.3).

Cada persona pertenece a un sistema en el que adquiere lenguaje y aprende a comunicarse con otros, Bertalanffy (1987), considera al sistema como el conjunto de elementos en interacción recíproca con el entorno. El sistema entabla relaciones dinámicas con otros sistemas a partir del manejo de la información necesaria para su desarrollo y la propensión a tomar decisiones, condiciones que determinan el modo como cada grupo opera en lo social a través del lenguaje, en este sentido Bronfrennbrenner (1987) a partir de la teoría ecológica platea que la familia es el sistema que define y establece en mayor medida el desarrollo de la persona desde su concepción, constituyéndose en el ámbito ecológico de desarrollo desde el nacimiento, pues en él se determina la conducta humana a razón del contexto sociocultural y el lenguaje que lo prescribe. Para este autor el entorno es algo que trasciende la situación inmediata y afecta directamente a la persona en desarrollo, por esta razón las familias que presentan vulnerabilidades a nivel interno y externo, al encontrarse en entornos peligrosos, tener antecedentes de violencia intrafamiliar, vivir escenarios excluyentes o presentar factores de riesgo real o potencial para sus miembros, tienen un mayor riesgo de presentar disfuncionalidades, mismas que pueden ser reforzadas debido al aumento del estrés ambiental y las necesidades insatisfechas a todo nivel.

La teoría ecológica considera que el principal microsistema es la familia, así, para su respectivo análisis se debe tener en cuenta la disponibilidad de cada miembro para hacer frente a las exigencias y demandas de los otros sistemas con los cuales se relaciona el grupo; en la familia se estudia los elementos estructurales, patrones de interacción familiar como también la historia personal de cada uno de sus integrantes (exosistema) mismos que reciben la influencia externa que pueden constituirse en factores de riesgo para la familia, tales como el estrés por consumo de bienes y servicios, problemas económicos, diferencias grupales y exclusión, desempeño y aislamiento social entre otros. Los niveles de análisis de los grupos incluyen la dinámica del mesosistema, el cual es el conjunto de sistemas con los que la familia guarda relación y con los que mantiene intercambios directos, es una dimensión importante en los informes de valoración familiar (Martins, C. 2007, pp.894-894); el mesosistema recibe la influencia de la familia, por lo que las tensiones familiares se reflejan en comportamientos externos que van desde la pasividad hasta la hostilidada y la apatía. El macrosistema es entendido como el conjunto de valores culturales, ideologías, creencias y políticas, da representación de la organización de las instituciones sociales. 
Para el modelo familiar sistémico los "sistemas familiares" representan a miembros de una familia mancomunados en una red a través de papeles, posiciones, valores y normas interdependientes, por ello lo que hace un individuo afecta de manera directa al resto sistema familiar (Foley. V, 1990) así, cada persona se encarga de desarrollar un rol asignado en la familia para que ésta funcione de manera adecuada. Para Virginia Satir (Bandler \& Grinder 1979, Satir 1967) la comunicación clara y directa es importante para el desarrollo saludable del sistema familiar así, una persona o familia disfuncional resulta ser el reflejo de un sistema familiar tenso o conflictivo; por otra parte Minuchin (1986) considera a la familia como un conjunto organizado e interdependiente de personas en constante interacción, regulado por reglas y funciones dinámicas internas y con el exterior, señala que los miembros de una familia se relacionan de acuerdo a reglas que constituyen la estructura familiar, misma que define como "el conjunto invisible de demandas funcionales que organizan los modos en que interactúan los miembros de una familia” (Rodrigo, 1998, p.86).

Los límites de las familias están compuestos por las reglas que precisan las personas que participan de las decisiones y la manera particular como lo hacen, por tal motivo tienen la función de proteger la especificidad del sistema, en este sentido los límites al interior del sistema permiten el establecimiento de las funciones individuales, responsabilidades, ejercicios y tareas de cada miembro con el fin de que la familia funcione adecuadamente (Dominici. 2003). Los límites al interior del sistema se establecen entre los subsistemas familiares (individual, conyugal, parental y fraterno) y pueden ser de tres tipos: los limites claros que definen las reglas de interacción con precisión; los límites difusos que no definen las reglas de interacción con precisión y caracterizan a las familias con personas muy dependientes y los limites muy Rígidos que definen interacciones en las que los miembros de la familia son independientes y sus mecanismos de apoyo se activan solo cuando existe un nivel muy alto de estrés (Minuchin \& Fischman, 1984; Haley, 1967). Es importante mencionar que los límites al exterior del sistema implican reglas de interacción entre la familia y otros sistemas, condición que en familias disfuncionales resulta compleja por la escasa interacción que presentan con el entorno.

\section{Metodologia.}

El estudio es de tipo cuantitativo descriptivo con un diseño descriptivo transversal. Analizado bajo el paradigma sistémico - familiar; en él se compararon los resultados estadísticos del APGAR familiar (instrumento de análisis) con las posturas teóricas del enfoque familiar sistémico, tomando en cuenta los indicadores de disfuncionalidad familiar y las derivaciones psicosociales que el hecho implica. Para la valoración de las familias se aplico la prueba APGAR familiar el cual consta de cinco puntos o variables y fue diseñado por Gabriel Smilkstein (1978) en la Universidad de Washington, (Alegre, P \& Suárez, M. RAMPA. 2006 1978). Estas variables son: la adaptación, participación, crecimiento, afecto y utilización de recursos de los miembros de la familia permitiendo un acercamiento a la realidad interna de las relaciones del sistema. Los puntos de corte son los siguientes: Buena función familiar (18 - 20); Disfunción familiar leve (14 - 17); Disfunción familiar moderada (10 - 13); Disfunción familiar severa (9 o menos). 


\section{Participantes.}

La muestra o población seleccionada corresponde a veinte mujeres que son casos denunciados de violencia intrafamiliar de la comisaria segunda del municipio de Armenia (Colombia), pertenecientes a las comunas tres, cuatro, cinco y seis, las cuales son especialmente afectadas por la violencia física y psicológica (PDD 2008; Andrade, J. 2010). La edad de la población oscila entre los 27 y 70 años, de estado civil variado (solteras, casadas, unión libre y separadas).

Procedimiento.

Para la realización del trabajo (encuesta, contacto comunitario y aplicación del instrumento) se precisó de la autorización de las mujeres afectadas por el maltrato y la aprobación de la comisaria segunda de familia del municipio de Armenia. El tiempo de aplicación del instrumento fue de 15 minutos. La muestra fue seleccionada a partir de un sondeo y entrevista estructurada la cual tuvo un tiempo de aplicación de 10 minutos. Los datos se tabularon en el programa Excel de Microsoft office de Windows.

\section{Resultados.}

De acuerdo a los resultados derivados a las variables y el instrumento empleado, se halló que en las mujeres entrevistadas prima el matrimonio (35\%) y la unión libre $(30 \%)$, un $20 \%$ son solteras, el 10\% están separadas y un 5\% divorciada. Respecto a la ocupación el 45\% son amas de casa, un 30\% tiene un empleo y el 25\% está desempleada. El estrato socioeconómico promedio es de 2 (35\%), el estrato 3 corresponde al $45 \%$, el 1 al 35\% estrato 4 fue de un $5 \%$. La edad promedio en que se presentan la mayoría de casos es de 30 años (30\%). De los 20 casos se tuvieron en cuenta el número de hijos para lo cual el número promedio de hijos es de 2 que corresponde a un $85 \%$ de las mujeres, un $5 \%$ no tiene hijos, el 35\% solo tienen un hijo, el 10\% de ellas tiene tres hijos y un $5 \%$ han tenido cuatro hijos. El promedio del puntaje de disfunción familiar en el cual se registraron la mayoría de los casos de violencia intrafamiliar fue de 12,6 o disfunción familiar grave y moderada (55\%), obteniendo un dato repetitivo con dos valores 12 y 16, que corresponde a un 15\% de la población. El tipo de violencia predominante es el maltrato físico (55\%) y psicológico (45\%). El estudio encontró que las familias presentan disfunción leve (35\%), moderada $(30 \%)$ y grave $(20 \%)$, mientras un porcentaje muy bajo de las mujeres entrevistadas tiene una buena función familiar (15\%). La relación entre variables mostró una correlación entre adaptación y participación familiar del 99\% lo cual indica que los desajustes adaptativos guardan una relación directamente proporcional con la participación que se le da a la mujer en el escenario familiar y social.

\section{Discusión.}

Si bien el "modelo ecológico" se ajusta a la dinámica de análisis de los hallazgos puesto que, concibe que el microsistema más importante es la familia, se debe de tomar en cuenta que en ella se producen, reproducen y mantienen los esquemas de comportamiento que dan cuenta de la VIF como un fenómeno sociocultural, por lo que la familia logra fortalecer los procesos de protección de sus miembros, como también puede llegar a potenciar los riesgos si al interior del sistema la autorregulación implica la admisión de características comportamentales, no acordes con los procesos de normalización de las conductas familiares en el escenario de lo social, lo cual implicaría una autopoiesis no ajustada al contexto 
comunitario y por tanto la exclusión sociofamiliar. Para Maturana y Varela (1984) "la característica más peculiar de un sistema autopoiético es que se levanta por sus propios cordones y se constituye como distinto del medio circundante a través de su propia dinámica, de tal manera que ambas cosas son inseparables" (Maturana, H. Varela. 1984, p.28), persé este sistema con su identidad debe ajustar su praxis a las demandas de tres amos: el entorno, el sistema de relaciones internas, y las dinámicas lingüísticas y afectivas de sus miembros.

La violencia intrafamiliar implica una práctica de dominación y exclusión social que surge como prolongación de los modelos patriarcales al interior de la familia, que en muchas ocasiones tiende a autorregularse convirtiéndose en el mecanismo de canalización de la angustia del agresor y en gran medida del núcleo familiar, así, la violencia se concentra principalmente en las instituciones sociales y se prolonga a través de los sistemas de identificación comunitaria hacia la familia por medio del lenguaje, el encuentro social, los imaginarios colectivos, las actitudes y los ataques internos y externos que sufra el sistema familiar El estudio encontró que cuando las familias presentan sesgos de machismo, tienen escasos conocimientos de prevención en temas de educación sexual y reproductiva, prevención del consumo de sustancias psicoactivas, resolución de conflictos y no reciben la influencia de actores sociales preventivos, se tornan proclives a la disfuncionalidad, lo cual se suma a una deficiente integración socio cultural, lo que conlleva un aislamiento comunitario que induce a un deterioro progresivo del sistema familiar. De acuerdo a Bronfenbrenner (1987) estos sistemas se quedan sin información sobre recursos sociales reales o potenciales, y sin apoyos formales frente a las dificultades leve, condición que aumenta el estrés y los problemas familiares.

En el caso de la violencia intrafamiliar la cultura sobresaliente en el contexto sociocultural se vuelve un espacio para contrastar con la cultura de origen (Hutchinson, G. 1981) lo cual implica analizar la idea prevalente de poder y obediencia ligada a los valores educativos y culturales con los que se da continuidad a los roles acerca de la mujer y el hombre. La VIF derivada de la disfunción familiar presenta una elevada tendencia a establecer relaciones horizontales de corte patriarcal, que casi siempre desembocan en actos agresivos hacia mujeres, niños y niñas y, en algunas ocasiones hacia el adulto mayor. En esto el peso cultural resulta importante pues, para muchas personas victimarias la agresión es asociada a cierta forma de protección la cual es a menudo programada por el agresor y aceptada por el agredido al convertirla en una expresión (disfuncional) de amor. De acuerdo con Forensis (2010) la razón de la marcada diferencia entre victimas por género guarda relación con el sistema de relaciones de poder, las cuales emergen al interior de la familia y entre los sexos, llegando a ser aprendidas "desde todas las fuentes posibles de educación tanto formal como no formal, que enseñan a observar el universo en sólo dos formas posibles: blanco o negro, bueno o malo, fuerte o débil, etc." (p.102).

La disfunción familiar que se puede generar por la falta de interacción dentro y fuera del grupo familiar, lo cual se refleja en una inadecuada comunicación y en relaciones conflictivas con personas y grupos a modo de consecuencia ante la vulneración del rol, la intimidad y la identidad del otro. Bronfenbrenner (1997) plantea que es de suma importancia la relación que se establece dentro y fuera de la familia, pues permite la instauración de un proceso de desarrollo al interior de contextos como la escuela, la familia y la sociedad en general. Este desarrollo es importante porque alli se crean los primeros vínculos afectivos, mismos que repercuten en la conducta del individuo a lo largo del ciclo vital; lo anterior se evidencia en algunas mujeres víctimas de violencia intrafamiliar que conviven con sujetos agresores, 
creando relaciones interpersonales disfuncionales e insatisfactorias, dado que un ambiente conflictivo afecta directamente el desarrollo individual y prosocial del individuo y el grupo. Según Andrade. J y Valencia. L (2011) lo anterior guarda relación, con el modo como la cultura influye en el desarrollo de la conducta ya que, cuando la mujer crece en espacios de verticalidad patriarcal, se establece un sesgo sobre sus roles (de crianza de hijos, cuidado de ancianos etc.) y su opinión suele ser muy poco valorada llegando a ser violentada a través del silenciamiento, la fuerza física, el abuso sexual a con actos de violencia sobre ella o sus hijos.

El primer entorno donde el sujeto aprende a relacionarse es la familia como escenario primario de socialización, sin embargo la familia recibe la influencia directa de un medio externo en el que se efectúa la interacción social a través de la comunicación y el lenguaje que afectan de forma indirecta las relaciones en el núcleo familiar. Mucha de la información recibida es ambivalente, negativa o proviene del peso cultural del imaginario social adscrito al rol y a la noción de masculinidad, lo cual genera factores de riesgo tales como, el estrés a todo nivel, la violencia en todas sus manifestaciones, frustraciones mayores en agresores y agredidos, temor, aislamiento social, derivación de la ansiedad en conductas de maltrato infantil, entre otros; de acuerdo con Andrade (2010 (1)) uno de los factores que impacta las dinámicas de los agresores, tiene que ver con una especie de sistema defensivo interno que se dispara de acuerdo a dos puntos básicos: la legitimación a través del "derecho maniqueista" de la condición de abuso del otro, el cual es justificado por el agresor, a través del recurso del legado generacional y la necesidad de compensación de intensas heridas del pasado, que se anudan al precario manejo de la culpa después del acto de violencia, lo anterior implica ver el fenómeno de la violencia intrafamiliar a partir de lo consecuente (consecuencias directas en el sistema) y lo causal de la actividad de los actores sociales implicados en la reproducción del fenómeno.

La disfunción familiar conlleva a que el núcleo familiar genere comportamientos y conductas inapropiadas respecto al modo de demostrar y expresar el afecto, apoyarse en nuevas actividades, compartir un tiempo diferente al de trabajar, solucionar conflictos, y permitir la participación en las decisiones familiares. De acuerdo con Ignacio Martín Baró (1988) para Kats (1960) "las actitudes pueden cumplir cuatro funciones: una función utilitaria de adaptación, una de defensa del yo contra los peligros externos y contra los conflictos internos, una función expresiva de los valores personales para afirmar la propia identidad, y una función cognoscitiva respecto al medio" (p.255), de acuerdo a esto la familia disfuncional puede verse afectada en una o en varias de estas funciones situación que se alimenta del peso ideológico de la actitud de muchas mujeres respecto al maltrato (no denuncia, tolera maltratos, los justifica), actitud que implica a menudo pasividad y ajustes inadaptativos. Los cambios biopsicosociales en las mujeres violentadas son evidentes, y se ven reforzados por la continuidad de la conflictividad de sus sistemas familiares lo cual repercute negativamente en la vida cotidiana y el desarrollo emocional de toda la familia.

Minuchin (1967) afirma que la estructura familiar es "el conjunto invisible de demandas funcionales que organizan los modos en que interactúan los miembros de una familia" (p.86), así, la familia se presenta como un conjunto de personas en constante interacción regidas por reglas y funciones. La disfuncionalidad familiar aguarda relación con el sistema de reglas o limites en el hogar las cuales están caracterizados por normas unilaterales que invierten la función de protección de sus miembros y aumentan los factores de riesgo respecto a la disolución de familia; estos límites se presentan a menudo en familias extensas con antecedentes de maltrato; Minuchin plantea que este tipo de familia llegan a los límites 
extremos y son caracterizados por la dependencia emocional que limita la autonomía, así, en las familias disfuncionales la verticalidad de la comunicación afecta el nivel de participación y la autoestima de la mujer maltratada llegando a reaccionar de forma exagerada ante cualquier situación (hipersensibilidad a las señales de violencia) y con una escasa capacidad de adecuación y reacomodación positiva respecto a la toma de decisiones. La mujer maltratada sufre cambios a nivel emocional, físico y social que ocasionan una mala acomodación a nuevos sistemas y a niveles de comunicación afectiva de tipo normofuncional.

Las familias nucleares son caracterizadas socialmente por el adecuado funcionamiento, sin embargo la investigación indica que estas familias cuando presentan antecedentes de violencia intrafamiliar pueden llegar a deteriorar su cohesión interna, situación que emerge de acuerdo a la percepción subjetiva de cada miembro y la experiencia de cada individuo con los actos de violencia. Las familias nucleares evaluadas están tipificadas como familias desligadas y cerradas, mismas que de acuerdo con Minuchin (1967) son independientes, con problemas de comunicación y se apoyan solamente cuando las situaciones de estrés llegan a un punto crítico. Para Virginia Satir (1986, citado por Andrade \& Valencia, 2011) los sistemas cerrados son aquellos, en que todos los miembros deben tener moderación para expresarse, en ellas no se permite la sinceridad porque se considera anormal; en este sentido la dificultad para comunicar el estado emocional en las familias disfuncionales de tipo nuclear genera incongruencias en la transmisión de los mensajes, mismos que son interpretados de manera inadecuada porque no queda clara su intencionalidad; en los casos de violencia intrafamiliar esto es un generador de incompatibilidad y distanciamiento emocional lo cual afecta la funcionalidad y la tendencia proactiva y autopoiética en las familias.

De acuerdo a los hallazgos la adaptación de las mujeres se ha visto afectada por la VIF pues los recursos intra y extra familiares para la resolución de los problemas resultan insuficientes, por lo que el equilibrio familiar se rompe continuamente y de manera especial en situaciones de tensión, lo que genera al interior del sistema un estado de crisis constante. Las mujeres víctimas de violencia presentan una desadaptación importante a un núcleo familiar en el que los integrantes no se apoyan mutuamente, lo cual constituye una condición muy relacionada a la escasa participación brindada a la mujer, lo cual no instaura la idea de solidaridad y cooperación en los integrantes de la familia, afectando directamente la toma de decisiones, la resolución de conflictos y la división del trabajo entre otros. El estudio evidencia que el crecimiento físico, emocional y social de la mujer se ve diezmado por el escaso apoyo mutuo, puesto que la inestabilidad de los sistemas coarta la capacidad emocional y social para tomar decisiones y crecer "a través y en" la las relaciones sociales. Las dificultades de apoyo y de sostén emocional por parte de su familia, afectan la participación y la expresión psicoafectiva, condición que modifica la capacidad para transmitir emociones, pensamientos, sentimientos y pasiones, generando temor y resentimientos en los afectados.

\section{Conclusiones y recomendaciones.}

La violencia intrafamiliar deteriora el tejido socio familiar afectando drásticamente el sistema de comunicación intra e intercomunitario, lo anterior tiene efectos graves en el desarrollo emocional y social de cada miembro familiar, por lo que la tendencia reaccionar de manera agresiva depende de variables cuyo peso emocional y cultural advierte sobre la prolongación generacional del fenómeno, razón por la que es importante estudiar el modo como los sistemas pueden autorregulare y el tipo de información preventiva necesaria para evitar estos 
eventos. La prevención es un proceso constante que empieza desde el hogar, se refuerza en otras instituciones a través de la comunicación y se reproduce en los diferentes escenarios de socialización, adscribiéndose a todos los sistemas de representación social, por tal motivo es en estos lugares donde se debe fortalecer su proceso, llegando a formar parte de la cotidianidad del encuentro, en este sentido se legitima el otro no en función de su vulnerabilidad sino a partir de su condición humana de inalienabilidad.

La disfuncionalidad familiar es el correlato del mal funcionamiento de otros sistemas sociales, los cuales chocan con el tipo de exceptivas y motivaciones de los grupos afectados por el fenómeno de la violencia y la exclusión social. Si bien la violencia intrafamiliar se presenta en todos los estratos socioeconómicos, es claro que aquellos colectivos sociales deprivados social y económicamente de los beneficios del estado social democrático y de derecho, se encuentran proclives a generar actos de violencia en los que se anula al otro al no reconocerlo como legítimo otro en la relación de convivencia (Maturana, 1991). La sociedad debe actuar de manera conjunta apoyando a las instituciones que intervienen a las madres violentadas, desarrollando y fortaleciendo los factores protectores de la violencia intrafamiliar al interior de los hogares, así la resignificación de patrones educativos en cuanto relación de pareja y buen trato a los hijos, propiciaría una mejor resiliencia además de la disminuir los índices de conflicto intrafamiliar, lo cual desemboca positivamente en un mejor funcionamiento de la familia.

La disfuncionalidad familiar tiene una relación directamente proporcional con experiencias de violencia intrafamiliar de las cuales las mujeres han sido históricamente victimas mayoritarias y aunque dicha relación no se constituya en la relación sine qua non es importante considerar que agresores, agredidos y espectadores sin un proceso de intervención psicosocial presentan una elevada tendencia a reproducir elementos de los que fueron víctimas, ya sea desde el punto de vista de la pasividad (inercia y entumecimiento ante el maltrato) como desde la actividad multiplicadora de la violencia. De acuerdo a lo expuesto los programas de intervención deben estar direccionados al desarrollo de habilidades preventivas en las familias a fin de evitar los legados generacionales de dolor y silenciamiento intrafamiliar, desde este orden de acciones es la familia la encargada de generar los recursos con los que autorregula sus sistema de representaciones y da cuenta de un mejor estado de la relaciones comunicacionales la interior del sistema. 


\section{Referencias Bibliográficas.}

ACNUR. (2008). Informe anual: Alta Comisionada de las Naciones Unidas para los Derechos Humanos sobre la situación de los derechos humanos en Colombia. A/HRC/10/032.

Alegre Palomino, Y. y Suárez M. RAMPA (2006). Instrumentos de Atención a la Familia: El Familiograma y el APGAR familiar: (1) 48-57. www.idefiperu.org/rampa.html.

Andrade, J. (2010 (1)). El maltrato familiar y el escenario mental del agresor. En: Colombia Revista Electronica De Psicologia Cientifica ISSN: 2011-2521 ed: Psicom Editores v.n/A fasc.N/A p.1 - 3, consultado el 20 de agosto de 2011 desde, http:/ / www.psicologiacientifica.com/bv/psicologia-431-1-el-maltrato-familiar-y-elescenario-mental-del-agresor.html.

Andrade, J. (2010). Pastoral social de Armenia. Programa ISEP. Informe final de actividades psicosociales. Junio de 2010. Documento institucional.

Andrade, J. Valencia, L (2011). Descripción de la Función familiar de 20 mujeres víctimas de violencia intrafamiliar psicológica de la comuna 4 del municipio de Armenia en el 2010. Colombia. Revista Aletheia. Corporación Universitaria Empresarial Alexander Von Humboldt. Ed: v. 1 fasc.N/A p.53-72.

Ares P. (1990) Mi familia es así. La Habana Cuba: Editorial de Ciencias Sociales.

Balbin , J., \& Insuasty Rodriguez, A. (2010). Las Victimas en Contextos de Violencia e Impunidad: Caso Medellín. Medellín: Instituto Popular de Capacticación.

Balbin, J., \& Insuasty Rodriguez, A. (2009). Victimas, Violencia y Despojo. Medellín: Litoimpacto.

Bandler y grinder 1976; Satir, 1967. Comportamiento anormal p.p.87.

Baro, M. (1988). “Acción e Ideología, psicología social”. UCA Editores. Salvador.

Bastidas, W., \& Insuasty Rodriguez, A. (2010). Victimas en Medellín. EL AGORA USB, 367$397,10(2)$

Bertalanffy, L. V. (1987) Teoría general de los sistemas. México: FCE.

Bronfennbrenner, U. (1987). La ecología del Desarrollo Humano, Barcelona, Paidos.

Defensoría del Pueblo de la República de Colombia. (2010). Comité de Prevención de Violencia Intrafamiliar y Abuso Sexual en el departamento del Quindío. estadística unificada de delitos del departamento. Consultado el 20 de febrero de 2011 desde, http:/ / www.portalfio.org/inicio/index.php?option=com_content\&view=article\&id= 515:colombia-se-reactiva-comite-de-prevencion-de-violencia-intrafamiliar-y-abusosexual-en-quindio\&catid=190\&Itemid $=400147$. 
Dominici L. (2003). "Terapia Familiar". Programa Salud de Adultos. Nivel Nacional. Primer Nivel de Atención. Guías de Atención. Caja del Seguro Social de Panamá. Diciembre del 2003.

Foley, V. Introdução à terapia familiar. Porto Alegre: Artes Médicas, 1990.

Forensis (2007). Instituto Nacional de Medicina Legal y Ciencias Forenses. Violencia intrafamiliar. Colombia. www.medicinalegal.gov.co/.

Forensis (2009). Violencia intrafamiliar. Colombia, Niños, niñas, adolescentes y mujeres, las víctimas de la violencia intrafamiliar. Disponible en, http:/ / www.medicinalegal.gov.co/index.php?option=com_content\&view=category\& $\mathrm{id}=19 \&$ Itemid $=55$.

Forensis (2010). VIOLENCIA INTRAFAMILIAR. COLOMBIA, Niños, niñas, adolescentes y mujeres, las víctimas de la violencia intrafamiliar. www.medicinalegal.gov.co/.

Franco, S. 1997. Revista panamericana de salud pública. Vol. 1, n² 2.

Gimeno, A. (1999). La familia: el desafio a la diversidad, Barcelona, Ariel.

http://www.who.int/mediacentre/news/releases/2005/pr62/es/index.html.

Hutchinson, G. Introducción a la ecología de poblaciones. (1981). Editorial blume, Barcelona

ICBF (2008). Bienestar familiar, congreso internación de violencia intrafamiliar. Revista memorias.

IMSS. (2003).Violencia contra la mujer: conocimiento y actitud del personal médico del Instituto Mexicano del Seguro Social, Morelos, México, salud publica México v 45.

Katz, D. (1960). The functional approach to the study of attitudes. Public Opinion Quarterly. 24: 163-204 (Trad. cast: El enfoque funcional en el estudio de las actitudes. En J. R. Torregrosay E. Crespo. Estudios Básicos de Psicología Social: 261-276. Barcelona, Morata).

Martins, C. (2007) la dinámica familiar en la visión de los padres y niños involucrados en violencia domestica contra infantes y adolescente. Revista latinoamericana enfermagem (online). Vol. 15, \# 5 .

Maturana, H. (1991). La democracia es una obra de arte. Mesa redonda magisterio. Instituto para la democracia Luis Carlos Galán. Bogotá.

Maturana. H, \& Varela, F. (1984). El árbol del conocimiento. Santiago de Chile, Editorial Universitaria.

Minuchin, S. (1984). Calidoscopio familiar Paidos. Buenos Aires, Barcelona.

Minuchin, S. (1986). Familias y terapia familiar. Barcelona, Gedisa. 
Minuchin, S. y Fishman, H. Ch. (1990). Técnicas de Terapia Familiar. México: Paidós.

PDD. (2008). Plan De Desarrollo Departamental "Quindío Unido". Julio Cesar López Espinosa Gobernador. Departamento Administrativo De Planeación, Dairo Enrique Valderrama Castro Director. Documento institucional.

Profamilia. (2005). Encuesta Nacional de Demografia y Salud. Profamilia.

Rodrigo, M. J. palacios J. (1998). familia y desarrollo humano.

Sagot, M. \& Carcedo, A. (2008). Program on Women, Health, and Development (Pan American Health Organization). Ruta critica de las mujeres afectadas por la violencia intrafamiliar en America Latina: estudios de caso de diez países.

Smilkstein, G. The family APGAR: (1978). a proposal for a family function test and its use by, physicians. J Fam Pract; 6: 1231-9.

Hurtado Domínguez, 2011, Yaqueline. (2011). estadísticas del comportamiento de delitos de abuso sexual y maltrato intrafamiliar En el Quindío, en: la crónica del Quindío. 